\title{
KIF18A promotes head and neck squamous cell carcinoma invasion and migration via activation of Akt signaling pathway
}

\author{
Guancheng Liu ${ }^{1,2}$, Gengming $\mathrm{Cai}^{3}$, Xiaosong $\mathrm{He}^{2}$, Donghai Huang ${ }^{1}$, Gangcai Zhu ${ }^{4}$, Changhan Chen ${ }^{1}$, \\ Xin Zhang ${ }^{1}$
}

${ }^{1}$ Department of Otolaryngology Head and Neck Surgery, Xiangya Hospital, Central South University, Changsha 410008, China; ${ }^{2}$ Department of Otolaryngology Head and Neck Surgery, Affiliated Hospital of Guilin Medical University, Guilin 541001, China; ${ }^{3}$ Department of Otolaryngology Head and Neck Surgery, First Affiliated Hospital of Quanzhou, Fujian Medical University, Quanzhou 362000, China; ${ }^{4}$ Department of Otolaryngology Head and Neck Surgery, The Second Xiangya Hospital, Central South University, Changsha 410010, China.

Contributions: (I) Conception and design: G Liu, X Zhang; (II) Administrative support: G Cai; (III) Provision of study materials or patients: X He; (IV) Collection and assembly of data: D Huang; (V) Data analysis and interpretation: G Zhu, C Chen; (VI) Manuscript writing: All authors; (VII) Final approval of manuscript: All authors.

Correspondence to: Xin Zhang. Department of Otolaryngology Head and Neck Surgery, Xiangya Hospital, Central South University, Changsha 410008, China. Email: xinzhang@csu.edu.cn.

Background: KIF18A has been shown to participate in the development of various human malignancies. However, the role of KIF18A in head and neck squamous cell carcinoma (HNSCC) remains unknown. This study investigated the function of KIF18A in HNSCC as well as its possible mechanisms.

Methods: In this study, we conducted in vitro experiments. First, we examined the effect of KIF18A on Tu686 and 6-10B cells via determining cell viability, colony formation ability and cell motility. And then, we examined that whether the carcinogenic effect of KIF18A is associated with Akt activation.

Results: Our current study demonstrated that KIF18A expression was increased in HNSCC patients and its cell lines. Knockdown and overexpression of KIF18A in HNSCC cells indicated that KIF18A promoted cancer cell proliferation, invasion and migration. Moreover, these bioactivity changes in HNSCC cells were accompanied by enhanced Vimentin expression and suppressed E-cadherin expression induced by KIF18A. Further mechanistic analysis revealed that the carcinogenic effect of KIF18A is associated with Akt activation, and blocking the activity of Akt reversed the malignant progression caused by KIF18A overexpression in HNSCC cells.

Conclusions: Together, our study reveals that KIF18A accelerates the progression of HNSCC and that targeting KIF18A may be a potential therapeutic strategy for the HNSCC.

Keywords: KIF18A; head and neck squamous cell carcinoma (HNSCC); proliferation; migration; invasion; Akt

Submitted Jun 19, 2019. Accepted for publication Sep 09, 2019.

doi: $10.21037 /$ tcr.2019.09.38

View this article at: http://dx.doi.org/10.21037/tcr.2019.09.38

\section{Introduction}

Head and neck squamous cell carcinoma (HNSCC) is the sixth most common cancer, accounting for more than $90 \%$ of all head and neck cancers with a global incidence of 550,000 cases per year (1). Although several improvements have been made in the diagnosis and treatment of patients with early stages of HNSCC, advanced stage HNSCC treatment results in poor outcomes due to infinite proliferation and metastasis $(2,3)$. Over the past few years, improvements in therapies contributing to cell proliferation and metastasis have been proposed to develop targeted agents for HNSCC therapy. Unfortunately, the life quality and survival rate of HNSCC patients have not significantly improved. Hence, discovering and fully understanding the key molecular mechanisms associated with tumorigenesis and progression are critical for improving the survival rate 
Table 1 Clinical features of the 27 patients

\begin{tabular}{lc}
\hline Variables & No. $(\%)$ \\
\hline Sex & $25(92.6)$ \\
Male & $2(7.4)$ \\
Female & \\
Age, years & $19(70.4)$ \\
$40-45$ & $8(29.6)$ \\
$>45$ & \\
AJCC clinical stage & $12(44.4)$ \\
I-II & $15(55.6)$ \\
III-IV & \\
T classification & $22(81.5)$ \\
T1-T2 & $5(18.5)$ \\
T3-T4 & \\
Lymph node metastasis & $11(40.7)$ \\
N- & $16(59.3)$ \\
N+ & \\
Distant metastasis & \\
M0 & \\
M1 & \\
\hline
\end{tabular}

for the HNSCC patients.

$\mathrm{KIF} 18 \mathrm{~A}$ is a member of the kinesin superfamily. It was first found to be associated with chromosome aggregation and centromere movement, through which chromosome stability and oscillation attenuation are maintained in mitotic spindle microtubules. Mutation and dysfunction of KIF18A can cause chromosome instability, leading to the pathological changes. In the recent years, mounting evidence has indicated that KIF18A is over-expressed in different types of tumors and play an important role in tumor genesis, aggressive and prognosis (4-6). KIF18A is over-expressed in human breast cancer; its high expression is associated with tumor grades, metastasis and poor survival, and may be a useful predictive marker for breast cancer (7-9). KIF18A is over-expressed in colorectal cancer cells (10), and it is significantly associated with tumor stage and metastasis; thus, KIF18A may be a significant factor for colorectal cancer progression (11). In human clear cell renal carcinoma, the level of KIF18A expression was significantly associated with tumor stage, histological grade, metastasis and tumor size (6). It has also been reported that high
KIF18A expressed promotes the proliferation, invasion and metastasis of HCC cells, correlates with an unfavorable prognosis and may emerge as a new target for the diagnosis and treatment of $\operatorname{HCC}(3,12)$.

KIF18A is often deregulated in many malignant tumors and plays an important role in cancer development and progression. However, the expression and the role of KIF18A in HNSCC are still unclear. No reports have elucidated the expression of KIF18A in HNSCC patients and its function in HNSCC cells. In the current study, KIF18A expression and its effects on proliferation and metastasis in human HNSCC were determined. KIF18A expression levels were compared in clinical specimens and normal adjacent tissues, and the effects of KIF18A on the proliferation, invasion and metastasis of HNSCC cells were determined by using the siRNA method. Moreover, we demonstrated that KIF18A may promote the proliferation, invasion and metastasis of HNSCC through the activity of Akt. KIF18A may serve as a potential prognostic marker for the patients with HNSCC and may play a critical role in the diagnosis and treatment of HNSCC.

\section{Methods}

\section{Patients and tissue preparation}

This study was conducted on a total of 27 head and neck tumour samples, which were histopathologically and clinically diagnosed at Xiangya Hospital, Central South University. For the use of these clinical materials for research purposes, prior consent was obtained from all patients, who provided written informed consent, and all research was performed in accordance with relevant guidelines. This study was approved by the Ethics Committee of the Xiangya Hospital of Central South University (ethics committee reference number: 201612679). The patients included 25 males and 2 females. None of the patients had a history of previous malignancies, radiotherapy or chemotherapy. The clinical information for and pathological characteristics of all patients are summarized in Table 1.

\section{Cell culture}

The HNSCC cell line Tu686 was kindly provided by Dr. Zhuo (Georgia) Chen; this cell line was harvested from a primary carcinoma located in the base of the tongue (13). The cells were cultured as previously described $(14,15)$. The 6-10B、5-8F were nasopharyngeal carcinoma (NPC) 
cell lines, which was established at the Central Experiment Laboratory of Xiangya Medical School, Central South University (Changsha, China). FaDu derived from human hypo-pharynx cancer was also friendly offered by Dr. Zhuo (Georgia) Chen. NP69 is immortalized non-malignant cell line derived from human nasopharyngeal mucosa.

We used a mixture containing RPMI-1640 medium, $10 \%$ fetal bovine serum (FBS), $100 \mathrm{IU} / \mathrm{mL}$ penicillin and $100 \mathrm{IU} / \mathrm{mL}$ streptomycin to culture the $6-10 \mathrm{~B}$ cells, and the cells were kept in a humidified atmosphere at $37{ }^{\circ} \mathrm{C}$ with $5 \% \mathrm{CO}_{2}$. Exponentially growing cells were used for all experiments. We used the Akt inhibitor LY294002 (Beyotime, China) to inhibit the expression of Akt at the concentration of $6 \mu \mathrm{g} / \mathrm{mL}$.

\section{Real-time PCR assay}

Total RNA was harvested from cells and tissues by TRIzol reagent following the manufacturer's directions (15596-018, Invitrogen) and was suspended with $20 \mu \mathrm{L}$ nuclease-free water. qRT-PCR was performed according to instrument of the qRT-PCR kit. Briefly, mRNAs was converted to cDNA using an All-in-One First Strand Synthesis Kit (GeneCopoeia, USA) according to the manufacturer's protocol. The primer sequences of KIF18A were determined using Primer Premier 5.0 software (Premier Biosoft, USA) and GAPDH was used as a control. The primer sequences for GAPDH were as follows: 5'-ATC AAG AAG GTG GTG AAG CAG3' (sense); and 5'-TGG AGG AGT GGG TGT CGC3' (antisense). The primer sequences for KIF18A were as follows: 5'-AAAAAGTGGTAGTTTGGGCTGA-3' (sense); and 5'-CTTTCAAGGGAGATGGCATTAG-3' (antisense). Products were amplified by PCR using Allin-One quantitative polymerase chain reaction (qPCR) mix (GeneCopoeia, USA), and the data were obtained and analyzed with the Applied Biosystems ViiA ${ }^{\mathrm{TM}} 7$ Real-Time PCR system. All RT reactions were performed in triplicate, and experimental procedures of $\mathrm{qPCR}$ were based on the manufacturer's guidelines. The relative expression levels were determined by the $2^{-\Delta \Delta \mathrm{Ct}}$ method.

\section{Western blotting}

Whole protein was harvested from the cultured cells using a protein extraction kit (Beyotime, China). A total of $30 \mu \mathrm{g}$ of protein was added to a $10 \%$ SDS-PAGE gel for separation and transfer onto PVDF membranes (Millipore).
Before adding primary antibodies, the membranes were blocked using TBST buffer supplemented with $1 \%$ bovine serum albumin at room temperature for $2 \mathrm{~h}$. After that the membranes were washed with TBST buffer 3 times and incubated with primary rabbit antibodies against KIF18A (ab72417, Abcam), p-Akt (Ser 473) (4060P, Cell Signaling Technology), Akt (4685S, Cell Signaling Technology), Vimentin (10366-1-AP, Proteintech), E-cadherin, (208741-AP, Proteintech), at $4{ }^{\circ} \mathrm{C}$ overnight. After washing, the membranes were incubated with the appropriate secondary antibody with HRP-labelled for $1 \mathrm{~h}$ at room temperature. Bands were finally visualized by employing the BeyoECL Plus Detection System (Beyotime, China). The protein expression levels were quantified by a FluorChem FC2 (San Leandro, CA, USA) and are represented as the densitometric ratio of the targeted protein to the housekeeping protein of GAPDH (Beyotime, China). The experiments were repeated three times.

\section{Small interfering RNA and cDNA transfection}

The KIF18A siRNA was obtained from RiboBio (RiboBio, China) to knock down KIF18A. The siRNA was transfected into cells using RNAi mate (GenePharma) according to the manufacturer's instruction. siRNAs' sequence was as follows: 5'-GUGCCACCAUAUGAAAGUATT-3' and 5'-UACUUUCAUAUGGUGGCACTT-3'. Vector (siRNA-NC):5 '-GAGUUAAAGUCAAAGUGACTT-3', 5'-GUCACUUUGACUUUAACUCTT-3'. The KIF18A cDNA was obtained from GeneCopoeia (EX-T1513-M98, GeneCopoeia): OmicsLink ${ }^{\mathrm{TM}}$ Expression Clone Datasheet of EX-T1513-M98; and the negative control (EXNEG-M98, GeneCopoeia): OmicsLink ${ }^{\mathrm{TM}}$ Expression Clone Datasheet of EX-NEG-M98. The target sequence for KIF18A and the negative control cDNA were transfected respectively. The cDNA was transfected into cells using Lipofectamine 2000 (Invitrogen Corporation) following the manufacturer's instructions.

\section{CCK-8 assay}

The proliferation of tumor cells was tested using a Cell Counting Kit-8 (Beyotime, China). A total of 4,000 cells were re-suspended in the complete medium and added to each well of a 96-well plate. After infection with KIF18A-siRNA or NC-siRNA for $48 \mathrm{~h}$, the cells were treated following the manufacturer's instructions. Then, the absorbance at $450 \mathrm{~nm}$ was detected by an ultraviolet 
spectrometer. The experiments were performed in quintuplicate and repeated in triplicate. The growth curves were generated using GraphPad Prism software.

\section{Colony formation assays}

Tu686 and 6-10B cells $\left(8 \times 10^{2}\right)$ suspended in RPMI- 1640 with $10 \%$ FBS were seeded in each well of a 6-well plate. The seeded cells received fresh culture medium every 2 days. After 10 days, the cells were fixed with paraformaldehyde and then stained with a $0.1 \%$ crystal violet solution. The experiments were performed in triplicate.

\section{Invasion and migration assay}

A wound healing assay was used to detect cell migration ability. When the treated cells formed a confluent monolayer in a 6-well plate well, a scratch was made on the monolayer by a sterile pipette tip $(10 \mu \mathrm{L})$, and the floating and detached cells were washed away using serum free medium. Finally, images were taken with an inverted fluorescence microscope at 0 and $48 \mathrm{~h}$. On each image, we randomly selected three sites to measure the width of the scratches, took the average value, the average scratch width w, and the Rate of Wound Healing (WHR) was calculated as $W H R=\left|\frac{\mathcal{W}_{0 h}-\mathcal{W}_{48 h}}{\mathcal{W}_{0}}\right| \times 100 \%$

Transwell assays using inserts precoated with Matrigel were used to test the cell invasion ability. Next, $4 \times 10^{3}$ treated cells suspended in $100 \mu \mathrm{L}$ of medium with $0.1 \%$ FBS were seeded on the culture inserts of a 24-well plate, and $400 \mu \mathrm{L}$ of medium containing $10 \%$ FBS was added to the lower as a chemotactic agent. After $48 \mathrm{~h}$, noninvasive cells located on the upper side of the membrane were wiped away, and cells on the other side off the membrane were treated with cold methanol $\left(-20^{\circ} \mathrm{C}\right)$, and then dried in air. Finally, the cells were stained with crystal violet and counted with an inverted microscope. The experiments were repeated in triplicate and 4 microscopic fields were counted per insert.

\section{Statistical analysis}

Statistical analyses were performed with SPSS software (version 17.0). The continuous variables in this research are expressed as the mean \pm SD. Significant differences between groups were determined with an analysis of variance (ANOVA). The chi-squared test was used for the statistical analysis of categorical variables. Differences were considered to be significant when $\mathrm{P}<0.05$. All tests were two-sided.

\section{Results}

\section{KIF18A is over-expressed in HNSCC}

To explore the potential associations between KIF18A expression and clinical variables in HNSCC patients, the expression levels of KIF18A mRNA were assayed by qPCR in 27 HNSCC patients. Our results revealed that expression levels of KIF18A mRNA were higher in the cancer tissues than in normal tissues (Figure 1A,B). Then we further investigated the expression of KIF18A mRNA in Tu686, 5-8f, 6-10B, CNE-2 and FADU cancer cell lines and the normal cell line NP69. We found that in the HNSCC cell lines of 5-8f, 6-10B, CNE-2, TU686 and FADU, KIF18A mRNA was expressed at higher levels than that in the human normal nasopharyngeal cell line NP69 (Figure 1C). Moreover, our western blot results showed that KIF18A expression of the 6-10B cells was the highest in the 6-10B, $5-8 \mathrm{~F}$ and Tu686 three cell lines, while it was the lowest in Tu686 cells (Figure 1D).

\section{Effect of KIF18A on the proliferation of SCCHN cells}

KIF18A have a important role in the proliferation of SCCHN cells (Figure 2). To directly determine whether KIF18A expression disrupts cell growth in HNSCC malignant progression, KIF18A was overexpressed in the HNSCC cell line Tu686. Our data revealed that forced expression of KIF18A correspondingly enhanced the proliferation (Figure $2 A$ ). The flat plate clones results showed that the number of Tu686 cells was increased nearly 2 fold in transfection with KIF18A cDNA cells compared with the negative control cDNA group (Figure $2 C, D$; $\mathrm{P}<0.01)$. We performed KIF18A loss-of-function analyses to strengthen the above results. The expression of KIF18A was down-regulated in 6-10B cells using siRNA technology. 6-10B cells were transfected with KIF18A siRNA expression vector or control siRNA expression vector. We found that KIF18A siRNA inhibited the proliferation of 6-10B cells in vitro (Figure $2 B$ ). Moreover, the expression of KIF18A decreased also effectively inhibited clone formation in 6-10B cells (Figure 2E,F). Together, these results indicated that KIF18A expression can promote the proliferation of HNSCC cancer cells, while silencing KIF18A expression inhibited cancer cell proliferation. It is not difficult to 


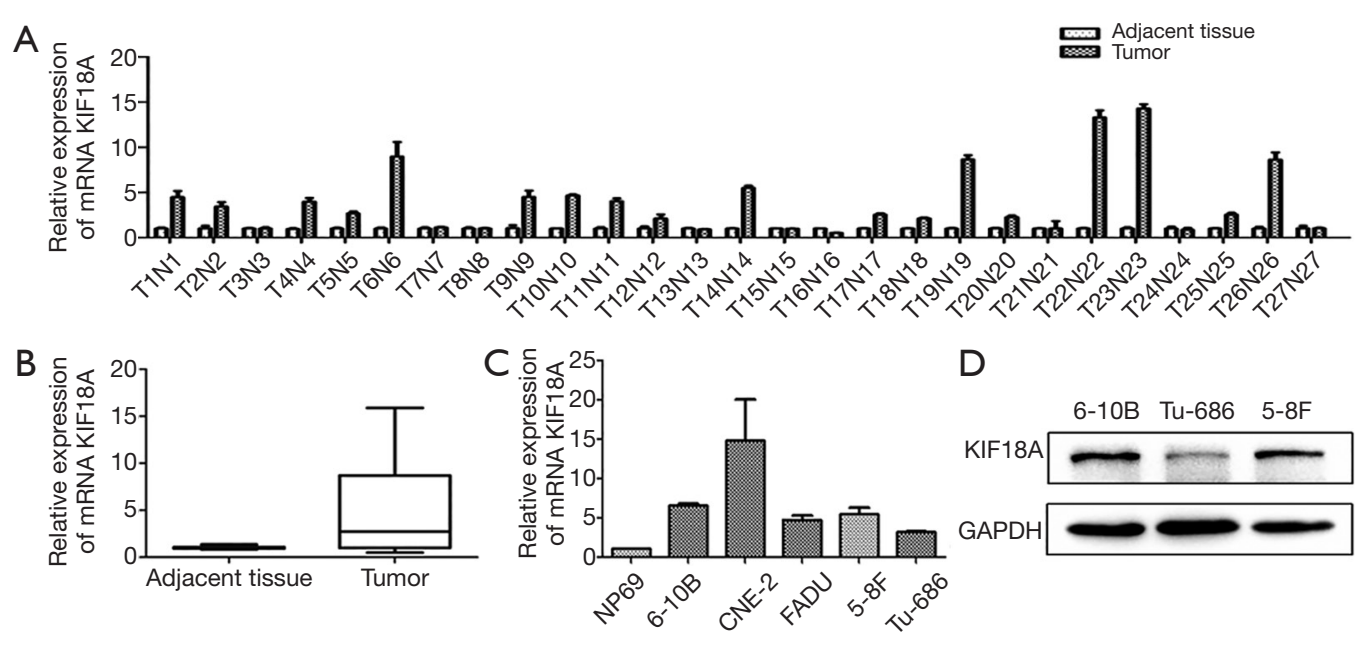

Figure 1 KIF18A is over-expressed in HNSCC. The expression of KIF18A in HNSCC tumors and normal tissues were analyzed by qPCR. $(\mathrm{A}, \mathrm{B}) \mathrm{KIF} 18 \mathrm{~A}$ mRNA expression were higher in HNSCC tissues than in the normal tissues; (C) KIF18A mRNA expression in the HNSCC cell lines Tu686, 5-8f, 6-10B, CNE-2 and FADU were significantly higher than that in the normal human normal cell line NP69; (D) the protein expression of Kif18A in HNSCC cell lines were determined by Western blot, lower in Tu686, and higher in 5-8F and 6-10B. HNSCC, head and neck squamous cell carcinoma. HNSCC, head and neck squamous cell carcinoma; qPCR, quantitative polymerase chain reaction.

infer that KIF18A may be an oncogene in HNSCC and a promoter of HNSCC malignant progression.

\section{KIF18A promotes the migration and invasion of HNSCC in vitro}

To further explore the effect of KIF18A on cell invasion and migration in vivo, the motility and invasiveness of 6-10B and Tu686 cells were evaluated by the cell scratch test and transwell chamber assay in this study. Tu686 cells were transfected with KIF18A vector or empty vector. The results of the cell scratch test demonstrated that the KIF18A increased the invasiveness of HNSCC cancer cells (Figure $3 A, B ; \mathrm{P}<0.01$ ). Following $48 \mathrm{~h}$ of cultured with the KIF18A vector, the number of Tu686 cells that invaded into the lower chamber markedly increased (Figure 3C,D; $\mathrm{P}<0.01)$. In contrast, when $6-10 \mathrm{~B}$ cells transfected with KIF18A-siRNA or NC-siRNA were cultured for $48 \mathrm{~h}$, the mean scratch distance between of 6-10B cells transfected with KIF18A siRNA was wider than that of the NC-siRNA group (Figure $3 E, F ; \mathrm{P}<0.01$ ). The transwell migration assay showed that the mean number of cells penetrating through the Matrigel down to the lower chamber was significantly lower in the 6-10B transfected KIF18A-siRNA group than in the blank group (Figure $3 G, H ; \mathrm{P}<0.01$ ).

Epithelial mesenchymal transformation (EMT) is an important manifestation of cancer. Tumors can be more invasive and metastatic through EMT. One important manifestation of the EMT is down-regulation of E-cadherin and up-regulation of Vimentin. And, our western blot results showed that KIF18A over-expressed in Tu686 cells could up-regulate Vimentin expression and down-regulate E-cadherin expression, which could greatly affect the invasion and migration abilities of tumor cells (Figure $4 A, B$; $\mathrm{P}<0.05)$. On the contrary, the expression of Vimentin was decreased and the expression of E-cadherin was increased by the downregulation KIF18A in 6-10B cells (Figure 4C,D; $\mathrm{P}<0.05)$. The above results indicated that the invasive and migration ability was significantly increased by KIF18A in HNSCC cells.

\section{Inbibition Akt attenuates the ability of KIF18A to enhance invasion and migration in HNSCC cells in vitro}

Our results indicated that KIF18A promoted the invasion and migration of HNSCC cells. A previous study showed that Akt was involved in the metastasis of HNSCC. Therefore, we investigated whether KIF18A exerted a tumor-promoting effect on Akt. We found that when the expression of Akt was inhibited, the expression of KIF18A would be increased in Tu686 cells. Cell scratch test and Transwell chamber assay results showed that inhibition 


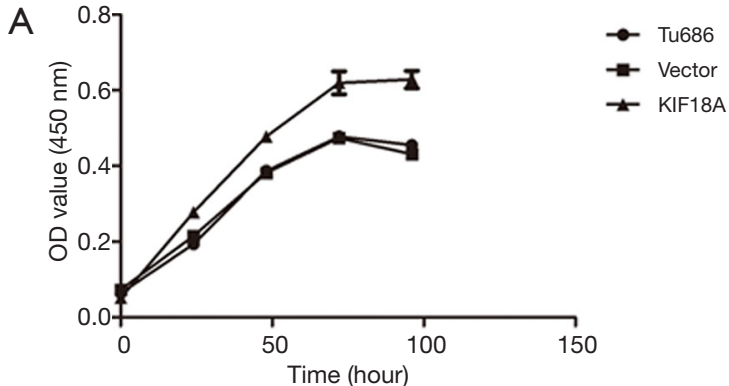

C
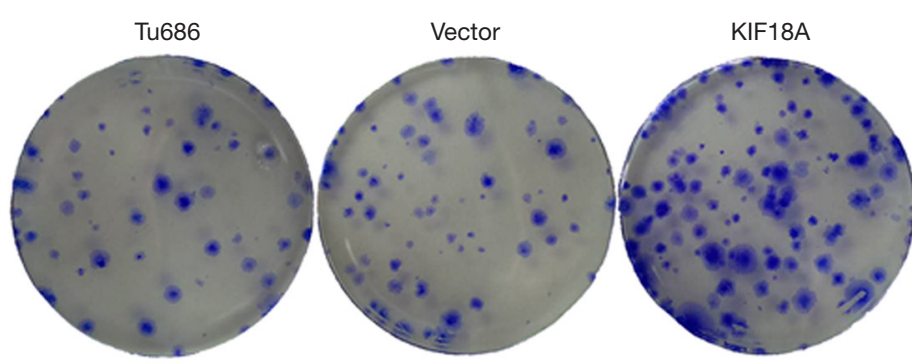

E

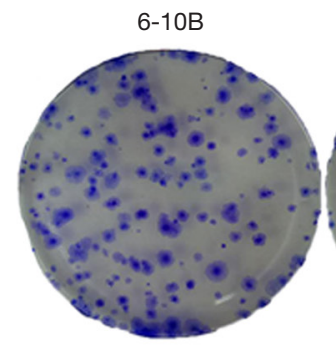

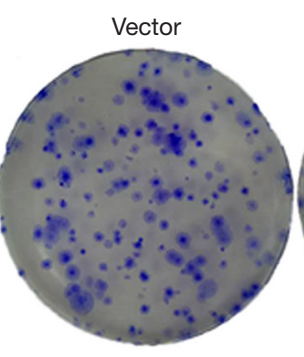

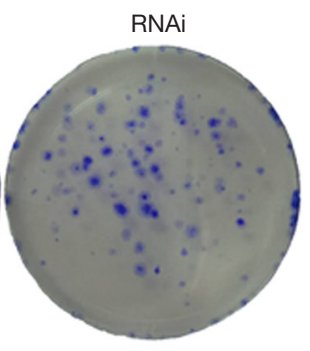

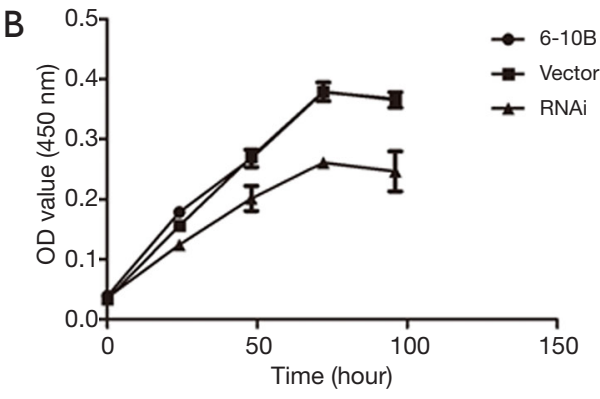
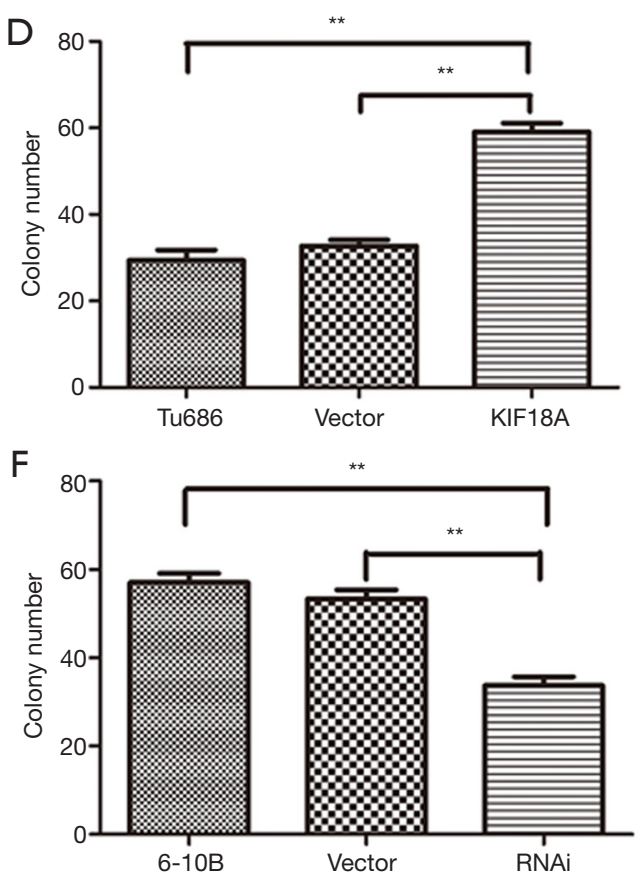

Figure 2 Effect of KIF18A on the proliferation of HNSCC cells were detected by CCK-8 and colony formation assay. (A) Up-regulated expression of KIF18A in Tu686 promoted cell proliferation; (B) down-regulated expression of KIF18A in 6-10B inhibited cell proliferation; (C,D) the results of the flat plate clones showed that the number of Tu686 cells were increased nearly 2-fold compared with the negative control cDNA group after transfection with KIF18A cDNA; (E,F) the expression of KIF18A decreased also effectively inhibited clone formation in $6-10 \mathrm{~B}$ cells. **, $\mathrm{P}<0.01$. HNSCC, head and neck squamous cell carcinoma. The staining method used in Figure $2 C, E$ is crystal violet staining.

of Akt could reverse the invasion and migration ability of KIF18A in HNSCC cells, indicating that KIF18A promotes tumor progression via a mechanism involving the Akt activation (Figure $5 A, B, C, D$ ). Our western blot results showed that Akt and p-Akt were up-regulated in KIF18A-overexpressed Tu686 cells and down-regulated in KIF18A-knocked down 6-10B cells (Figure 6A,B,C,D). To confirm the role of the Akt in KIF18A-mediated metastasis, the expression levels of E-cadherin and Vimentin were measured upon KIF18A over-expression with Akt inhibited Tu686 cells. As shown in Figure 6E, F, the up-regulation of
Vimentin and down-regulation of E-cadherin mediated by KIF18A were obviously attenuated by the inhibition of Akt. These results suggested that Akt activation was involved in the progression of HNSCC cells induced by KIF18A.

\section{Discussion}

HNSCC ranks as the sixth most aggressive malignancy in the world. Although multiple diagnosis and therapy methods have been improved significantly, the prognosis of HNSCC patients is still unsatisfactory for distant metastases 


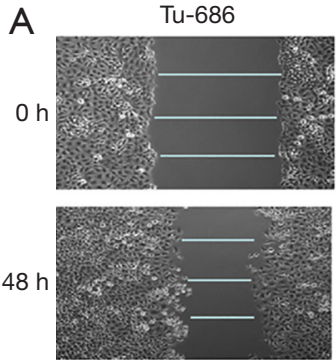

C

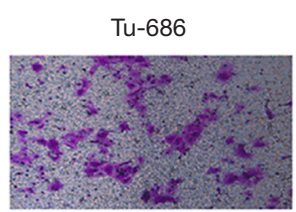

E
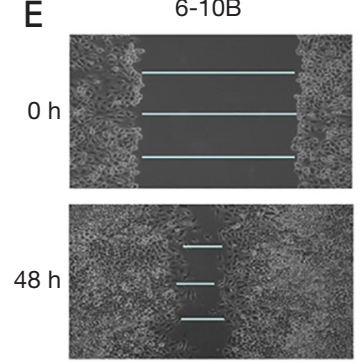

G

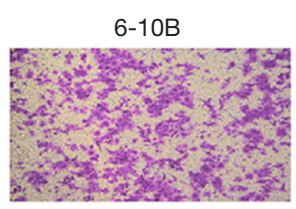

Vector
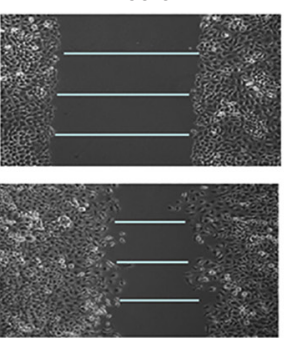

Vector

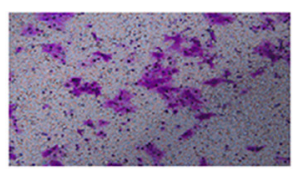

Vector
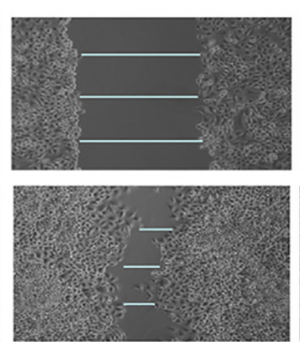

Vector

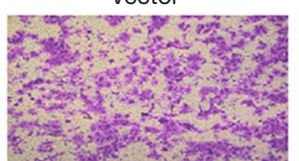

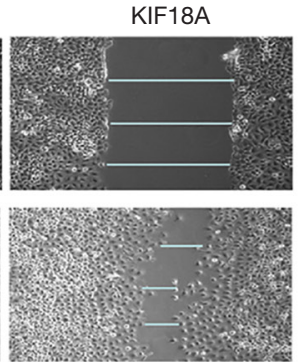

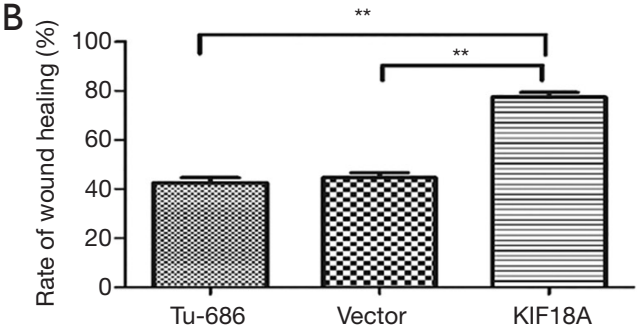

KIF18A

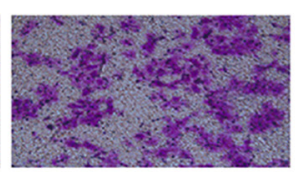

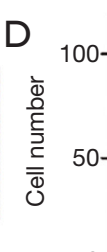

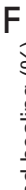

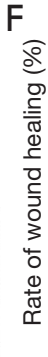
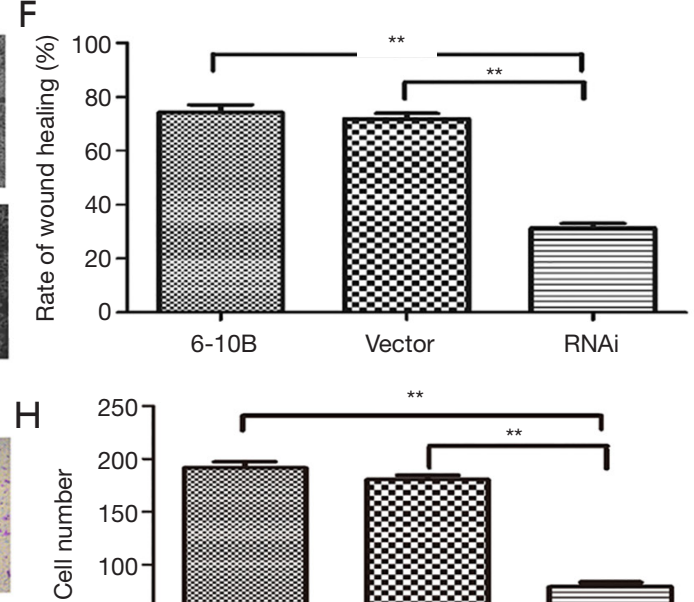

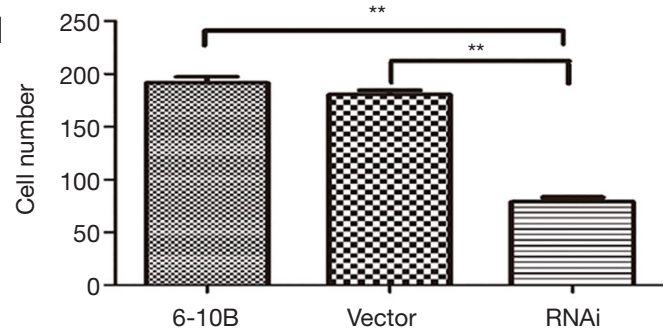

Figure 3 KIF18A promoted the invasion and migration abilities of HNSCC cells. (A,B) wound healing assay revealed that upregulated KIF18A expression promoted migration in Tu686 cells; (C,D) transwell analysis showed that upregulated KIF18A expression enhanced the invasion ability of Tu686 cells; (E,F) wound healing assays revealed that KIF18A knocked down inhibited 6-10B cells migration; (G,H) transwell analysis showed that downregulated KIF18A expression inhibited invasion in 6-10B cells. **, P $<0.01$. HNSCC, head and neck squamous cell carcinoma. The cell scale bar in Figure $3 E, G$ is $40 \times$.

(16-18). Therefore, it is urgent and imperative to clarify the mechanisms underlying metastasis, which will benefit future surveillance and targeted therapy for HNSCC patients. KIF18A, a member of the kinesin 8 family, is an important regulator that controls mitotic chromosome alignment during mitosis (19-22). However, recent studies report that KIF18A plays an important role in carcinogenesis and cancer progression. Experimental evidence indicates that KIF18A expression is significantly higher in cancer tissues than in paired normal tissues $(6,12,23)$, and its high expression is closely related to adverse prognostic factors and shorter survival times $(4,7,9,11)$. Recently studies found that KIF18A was involved in the invasion and metastasis processes of some malignant tumors, such as hepatocellular carcinoma, breast cancer, renal cell carcinoma and colorectal cancer. KIF18A can also be a significant factor for cancer progression. In HNSCC, however, the function of KIF18A and its role in metastasis have not been clearly elucidated. 

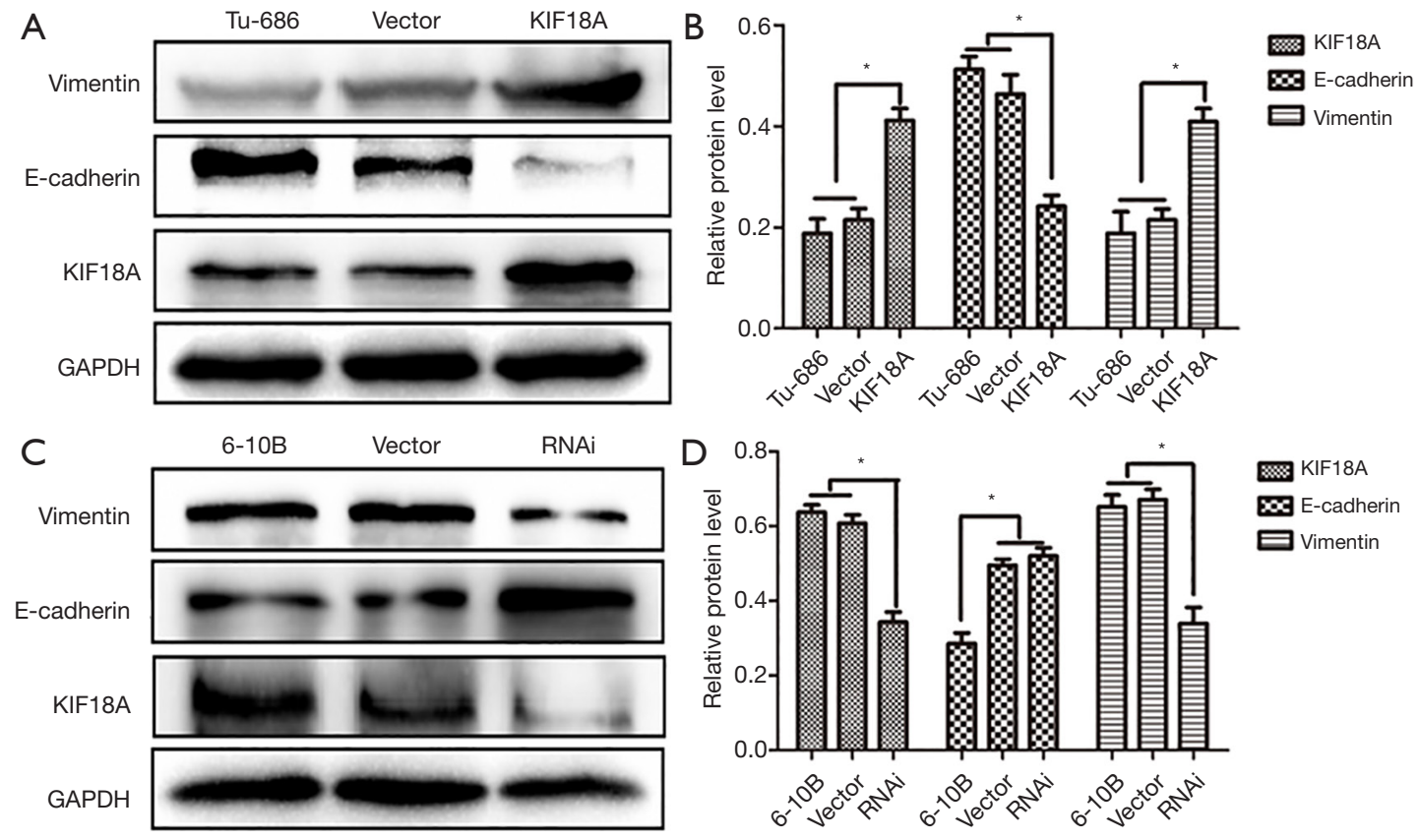

Figure 4 KIF18A can regulate the expression of EMT-related proteins. (A,B) The western blot experiment showed that KIF18Aoverexpressed in Tu686 cells could up-regulate Vimentin expression and down-regulate E-cadherin expression; (C,D) the expression of Vimentin was decreased and the expression of E-cadherin was increased by the down-regulated KIF18A in 6-10B cells. **, P <0.01.

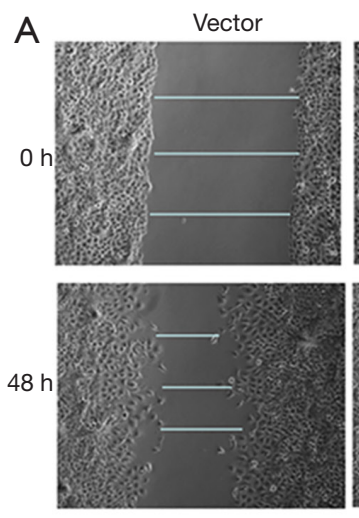

C Vector

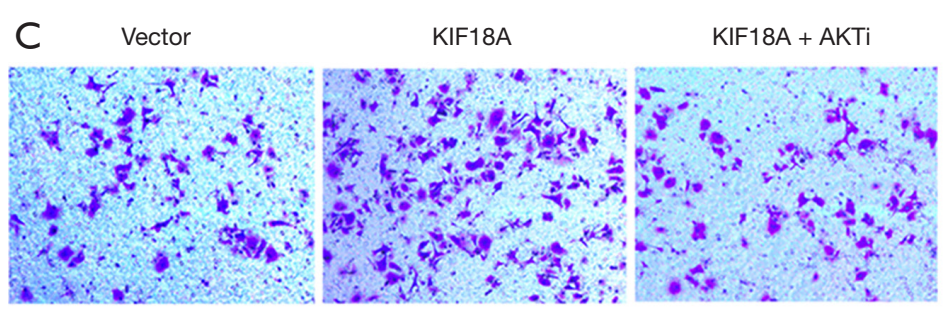

KIF18A
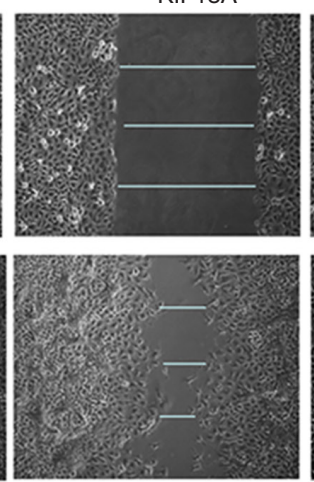

$\mathrm{KIF} 18 \mathrm{~A}+\mathrm{AKTi}$

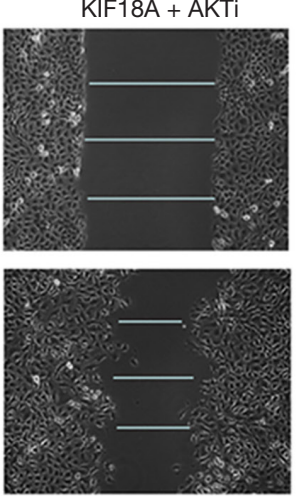

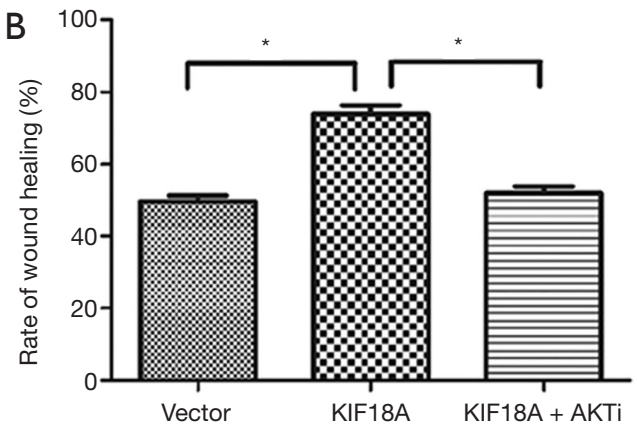

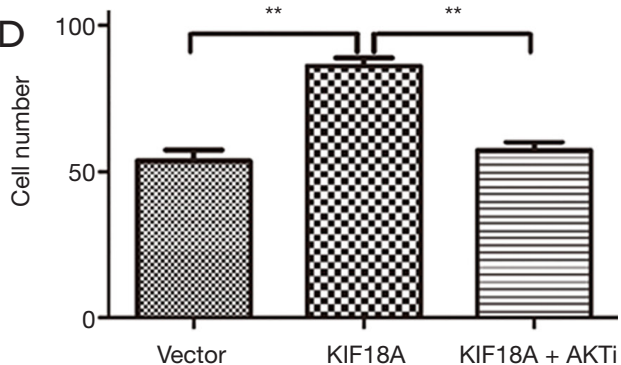

Figure 5 In Tu686 cells, up-regulated expression of KIF18A can promote cell migration, which can be reversed by AKT inhibitor. (A,B) Migration assays were used to detect the migration and invasion of Tu686 cells after transfected with KIF18A cDNA, KIF18A cDNA + AKTi; (C,D) invasion assays were used to detect the migration and invasion of Tu686 cells after transfected with KIF18A cDNA, KIF18A cDNA + AKTi. *, $\mathrm{P}<0.05 ;$ **, $\mathrm{P}<0.01$. The staining method used in Figure $5 C$ is crystal violet staining. 

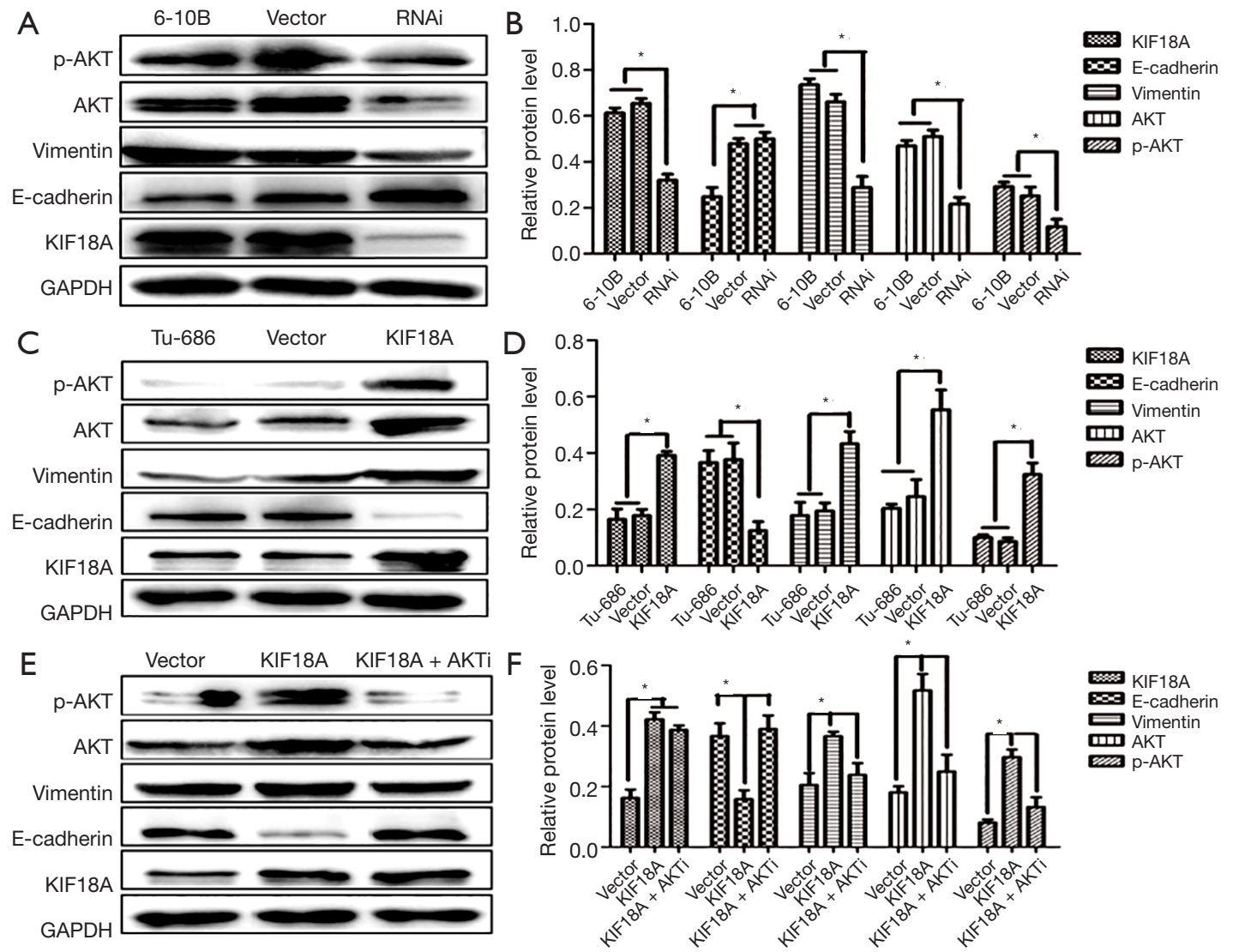

Figure 6 Kif18a regulates the expression of EMT-related proteins through Akt. (A,B) Down-regulated expression of KIF18A in 6-10B inhibited the protein expression of Vimentin, Akt and p-Akt, promoted the expression of E-cadherin; (C,D) up-regulated expression of KIF18A in Tu686 promoted the protein expression of Vimentin, Akt and p-Akt, inhibited the expression of E-cadherin; (E,F) in KIF18A upregulated Tu686 cells, Akt inhibitor can reversed the protein expression of vimentin and E-cadherin. * $\mathrm{P}<0.05$.

In this study, we found that KIF18A mRNA expression was obviously higher in HNSCC tissues than in adjacent tissues. Moreover, KIF18A was also found to be expressed at higher levels in HNSCC cancer cells than in normal cells, which may promote the progression of HNSCC. To further explore the function of KIF18A in the development of HNSCC cells, we over-expressed KIF18A in Tu686 cells and knocked down its expression in 6-10B cells to investigate the changes in cancer cell growth, invasion and migration. Our results showed that KIF18A overexpression promoted cell proliferation, cell invasion and migration in Tu686 cells. In contrast, after silencing KIF18A, cell proliferation, cell invasion and migration ability were decreased. These changes suggest that the KIF18A gene controls cell mitosis and promotes cell division, which may be involved in biological activity of cancer cells. This indicates that abnormal KIF18A expression in HNSCC may cause abnormal regulation of mitosis and caused cancer cell malignant hyperplasia, but the specific molecular mechanism through which KIF18A promotes metastasis remains uncertain.

Epithelial mesenchymal transformation is an important manifestation of cancer. Tumors can be more invasive and metastatic through epithelial mesenchymal transformation. One important manifestation of the is down-regulation of E-cadherin and up-regulation of Vimentin. For further understand the molecular mechanism of KIF18A in HNSCC, we detected the protein expression of Vimentin and E-cadherin, which are associated with the ability of invasion and migration in cancer cells. Vimentin is commonly expressed in mesenchymal cells and its abnormal expression is closely associated with a high degree of invasiveness and metastasis (24-26). The metastatic risk of high Vimentin expression was higher than that of low Vimentin expression 
in HNSCC patients (27). E-cadherin is a cell adhesion molecule and is expressed in the membrane of most epithelial cells (28). Available studies have investigated E-cadherin acted as a tumor suppressor in various epithelial tumors and its expression decreased or aberrant was related to cancer invasion and metastasis in HNSCC (27,29-31). Downregulated E-cadherin expression and up-regulated Vimentin expression enhance HNSCC cell migration (27). Our western blot results showed that KIF18A overexpression increased the expression of Vimentin and decreased the expression of E-cadherin in Tu686 cells. Conversely, KIF18A knockdown inhibited Vimentin expression and enhanced E-cadherin expression in 6-10B cells. These data indicated that KIF18A might enhance cell invasion and migration by regulating EMT.

It has been shown that the Akt signaling pathway was involved in the migratory and invasive bioactivity of cancer cells (32-35), including HNSCC cells (36). Activation of Akt could promote cancer cells metastasis $(32,35,37)$. Considering the role of KIF18A and Akt in the progression of HNSCC, so the expression of KIF18A may be associated with the Akt activation. In the KIF18A-overexpressing Tu686 cells, Akt expression inhibited could ablate the carcinogenic effect of KIF18A on HNSCC cells. Moreover, the activation of Akt inhibited in KIF18A-overexpressing Tu686 cells also effectively reversed the expression of Vimentin and E-cadherin which are regulated by KIF18A. Overall, our results shown that KIF18A can accelerate cell proliferation and metastasis via activating Akt.

\section{Conclusions}

This study confirmed that KIF18A was highly expressed in HNSCC patient tissues and cell lines. Enhanced KIF18A expression promoted cell proliferation, migration and invasion by regulating the expression of Vimentin and E-cadherin in HNSCC cells. And the effects of KIF18A accelerated HNSCC cell proliferation and metastasis were associated with the activation of Akt. Therefore, our findings imply that targeting KIF18A may be a potential treatment for HNSCC.

\section{Acknowledgments}

Funding: The study was supported by grants from the Natural Science Foundation of China (No. 81602389, 81472696, 81202128, 81660294, 81272974).

\section{Footnote}

Conflicts of Interest: The authors have completed the ICMJE uniform disclosure form (available at http://dx.doi. org/10.21037/tcr.2019.09.38). The authors have no conflicts of interest to declare.

Ethical Statement: The authors are accountable for all aspects of the work in ensuring that questions related to the accuracy or integrity of any part of the work are appropriately investigated and resolved. The study was conducted in accordance with the Declaration of Helsinki (as revised in 2013). All patients selected for our study were fully informed about our experimental protocols, which were approved by the Ethics Committee of the Xiangya Hospital of Central South University (ethics committee reference number: 201612679).

Open Access Statement: This is an Open Access article distributed in accordance with the Creative Commons Attribution-NonCommercial-NoDerivs 4.0 International License (CC BY-NC-ND 4.0), which permits the noncommercial replication and distribution of the article with the strict proviso that no changes or edits are made and the original work is properly cited (including links to both the formal publication through the relevant DOI and the license). See: https://creativecommons.org/licenses/by-nc-nd/4.0/.

\section{References}

1. Yu C, Wang Y, Li G, et al. LncRNA PVT1 promotes malignant progression in squamous cell carcinoma of the head and neck. J Cancer 2018;9:3593-602.

2. Shetty AV, Wong DJ. Systemic Treatment for Squamous Cell Carcinoma of the Head and Neck. Otolaryngol Clin North Am 2017;50:775-82.

3. Saloura V, Vougiouklakis T, Sievers C, et al. The role of protein methyltransferases as potential novel therapeutic targets in squamous cell carcinoma of the head and neck. Oral Oncol 2018;81:100-8.

4. Liao W, Huang G, Liao Y, et al. High KIF18A expression correlates with unfavorable prognosis in primary hepatocellular carcinoma. Oncotarget 2014;5:10271-9.

5. Zhu H, Xu W, Zhang H, et al. Targeted deletion of Kif18a protects from colitis-associated colorectal (CAC) tumors in mice through impairing Akt phosphorylation. Biochem Biophys Res Commun 2013;438:97-102. 
6. Chen QI, Cao B, Nan N, et al. Elevated expression of KIF18A enhances cell proliferation and predicts poor survival in human clear cell renal carcinoma. Exp Ther Med 2016;12:377-83.

7. Zhang C, Zhu C, Chen H, et al. Kif18A is involved in human breast carcinogenesis. Carcinogenesis 2010;31:1676-84.

8. Kasahara M, Nagahara M, Nakagawa T, et al. Clinicopathological relevance of kinesin family member $18 \mathrm{~A}$ expression in invasive breast cancer. Oncol Lett 2016;12:1909-14.

9. Alfarsi LH, Elansari R, Toss MS, et al. Kinesin family member-18A (KIF18A) is a predictive biomarker of poor benefit from endocrine therapy in early ER+ breast cancer. Breast Cancer Res Treat 2019;173:93-102.

10. Shichijo S, Ito M, Azuma K, et al. A unique gene having homology with the kinesin family member $18 \mathrm{~A}$ encodes a tumour-associated antigen recognised by cytotoxic $\mathrm{T}$ lymphocytes from HLA-A2+ colon cancer patients. Eur J Cancer 2005;41:1323-30.

11. Nagahara M, Nishida N, Iwatsuki M, et al. Kinesin 18A expression: clinical relevance to colorectal cancer progression. Int J Cancer 2011;129:2543-52.

12. Luo W, Liao M, Liao Y, et al. The role of kinesin KIF18A in the invasion and metastasis of hepatocellular carcinoma. World J Surg Oncol 2018;16:36.

13. Sacks PG. Cell, tissue and organ culture as in vitro models to study the biology of squamous cell carcinomas of the head and neck. Cancer Metastasis Rev 1996;15:27-51.

14. Yu C, Liu Y, Huang D, et al. TGF- $\beta 1$ mediates epithelial to mesenchymal transition via the TGF- $\beta /$ Smad pathway in squamous cell carcinoma of the head and neck. Oncol Rep 2011;25:1581-7.

15. Liu Y, Yu C, Qiu Y, et al. Downregulation of EphA2 expression suppresses the growth and metastasis in squamous-cell carcinoma of the head and neck in vitro and in vivo. J Cancer Res Clin Oncol 2012;138:195-202.

16. She L, Qin Y, Wang J, et al. Tumor-associated macrophages derived CCL18 promotes metastasis in squamous cell carcinoma of the head and neck. Cancer Cell Int 2018;18:120.

17. Garcia-Pedrero JM, Martinez-Camblor P, Diaz-Coto S, et al. Tumor programmed cell death ligand 1 expression correlates with nodal metastasis in patients with cutaneous squamous cell carcinoma of the head and neck. J Am Acad Dermatol 2017;77:527-33.

18. Pattje WJ, Melchers LJ, Slagter-Menkema L, et al. FADD expression is associated with regional and distant metastasis in squamous cell carcinoma of the head and neck. Histopathology 2013;63:263-70.

19. Yang F, Chen Y, Dai W. Sumoylation of Kif18A plays a role in regulating mitotic progression. BMC Cancer 2015;15:197.

20. Ye F, Tan L, Yang Q, et al. HURP regulates chromosome congression by modulating kinesin Kif18A function. Curr Biol 2011;21:1584-91.

21. Mayr MI, Hummer S, Bormann J, et al. The human kinesin Kif18A is a motile microtubule depolymerase essential for chromosome congression. Curr Biol 2007;17:488-98.

22. Stumpff J, von Dassow G, Wagenbach $M$, et al. The kinesin-8 motor Kif18A suppresses kinetochore movements to control mitotic chromosome alignment. Dev Cell 2008;14:252-62.

23. Wang L, Yang S, Sun R, et al. Expression of KIF18A in gastric cancer and its association with prognosis. Zhonghua Wei Chang Wai Ke Za Zhi 2016;19:585-9.

24. Semelakova M, Grauzam S, Betadthunga $P$, et al. Vimentin and Non-Muscle Myosin IIA are Members of the Neural Precursor Cell Expressed Developmentally Down-Regulated 9 (NEDD9) Interactome in Head and Neck Squamous Cell Carcinoma Cells. Transl Oncol 2019;12:49-61.

25. Dal Vechio AM, Giudice FS, Sperandio FF, et al. Vimentin expression and the influence of Matrigel in cell lines of head and neck squamous cell carcinoma. Braz Oral Res 2011;25:235-40.

26. Dong Y, Zheng Y, Wang C, et al. MiR-876-5p modulates head and neck squamous cell carcinoma metastasis and invasion by targeting vimentin. Cancer Cell Int 2018;18:121.

27. Nijkamp MM, Span PN, Hoogsteen IJ, et al. Expression of E-cadherin and vimentin correlates with metastasis formation in head and neck squamous cell carcinoma patients. Radiother Oncol 2011;99:344-8.

28. Georgolios A, Batistatou A, Manolopoulos L, et al. Role and expression patterns of E-cadherin in head and neck squamous cell carcinoma (HNSCC). J Exp Clin Cancer Res 2006;25:5-14.

29. Shergill K, Sen A, Pillai HJ. Role of E-cadherin and cyclin D1 as predictive markers of aggression and clonal expansion in head and neck squamous cell carcinoma. J Korean Assoc Oral Maxillofac Surg 2018;44:182-90.

30. Ren X, Wang J, Lin X, et al. E-cadherin expression and prognosis of head and neck squamous cell carcinoma: evidence from 19 published investigations. Onco Targets 
Ther 2016;9:2447-53.

31. Fujii R, Imanishi Y, Shibata K, et al. Restoration of E-cadherin expression by selective Cox-2 inhibition and the clinical relevance of the epithelial-to-mesenchymal transition in head and neck squamous cell carcinoma. J Exp Clin Cancer Res 2014;33:40.

32. Xu J, Zhou J, Dai H, et al. CHIP functions as an oncogene by promoting colorectal cancer metastasis via activation of MAPK and AKT signaling and suppression of E-cadherin. J Transl Med 2018;16:169.

33. Yang S, Ji Q, Chang B, et al. STC2 promotes head and neck squamous cell carcinoma metastasis through modulating the PI3K/AKT/Snail signaling. Oncotarget 2017;8:5976-91.

34. Liu L, Miao L, Liu Y, et al. S100A11 regulates renal

Cite this article as: Liu G, Cai G, He X, Huang D, Zhu G, Chen C, Zhang X. KIF18A promotes head and neck squamous cell carcinoma invasion and migration via activation of Akt signaling pathway. Transl Cancer Res 2019;8(6):2252-2263. doi: $10.21037 /$ tcr.2019.09.38 carcinoma cell proliferation, invasion, and migration via the EGFR/Akt signaling pathway and E-cadherin. Tumour Biol 2017;39: 1010428317705337.

35. Li A, Zhang W, Xia H, et al. Overexpression of CASS4 promotes invasion in non-small cell lung cancer by activating the AKT signaling pathway and inhibiting E-cadherin expression. Tumour Biol 2016;37:15157-64.

36. Yu C, Liu Y, Tan H, et al. Metadherin regulates metastasis of squamous cell carcinoma of the head and neck via AKT signalling pathway-mediated epithelial-mesenchymal transition. Cancer Lett 2014;343:258-67.

37. Lee WH, Choong LY, Jin TH, et al. TRPV4 plays a role in breast cancer cell migration via $\mathrm{Ca}$-dependent activation of AKT and downregulation of E-cadherin cell cortex protein. Oncogenesis 2017;6:e338. 